\title{
Der private Haushalt als Arbeitsplatzreservoir? Zur Akzeptanz und Abwehr von Haushaltsdienstleistungen
}

Wie stehen die Chancen, dass Dienstleistungen für Haushalte zu einem normalen Arbeitsmarktsegment werden? Die hier vorgestellten Befunde einer empirischen Studie verdeutlichen den Bedarf an und die Nachfrage nach Haushaltsdienstleistungen. Sie zeigen zugleich die weit verbreiteten Vorbehalte gegenüber Haushaltsdienstleistungen. Die genaue Analyse der Haushalte, die Dienstleistungen nutzen, beweist aber auch, dass mit Flexibilität, Pragmatismus und der Bereitschaft, die durch Haushaltsdienstleistungen erbrachten Leistungen anzuerkennen, ein Arbeitsmarkt entstehen könnte, in dem sowohl Qualitätsstandards gelten als auch soziale Mindeststandards gewährleistet sind.

\section{1 \\ Einleitung}

Im wohlfahrtsstaatlichen Dreieck StaatArbeitsmarkt-Familie sind Umfang und Ausmaß der Privatheit von Haushaltsarbeit und Kinderbetreuung ein wichtiges Element zur Unterscheidung verschiedener Wohlfahrtsregime. Wenn diese Aufgaben fast vollständig der Familie übertragen werden, dann „passt“ dazu ein Sozialsystem, das die Rollenkonstellation von Hausfrau und Familienernährer begünstigt. Die Arbeit im Haus ist gesellschaftlich unsichtbar, und Dienstleistungen für den Haushalt haben in diesem System keinen Stellenwert (Geissler 2010). Angesichts der seit den 1960er Jahren steigenden Bildungsund Erwerbsbeteiligung der Frauen und im Kontext des arbeits- und sozialpolitischen Paradigmenwechsels wird jedoch auch in Deutschland die Erwerbsarbeit von Frauen seit geraumer Zeit gefördert. Neues Leitbild ist der „adult worker“ (Lewis 2004); demnach soll jede und jeder Erwachsene kontinuierlich erwerbstätig sein. Die Durchsetzung dieses Postulats stößt jedoch an institutionelle, individuell-biografische wie auch alltagspraktische Grenzen. Zum einen sind in Deutschland bisher wichtige familienpolitische Regelungen nicht an die neue Arbeitsmarktdoktrin angepasst. Zum anderen sehen sich die Individuen weiterhin der Unverträglichkeit von erwerbszentrierter Lebensführung, Familienverantwortung und Haushaltsarbeit gegenüber. Aufgrund der Beschleunigung fast aller Lebensvollzüge und der damit zunehmenden Zeitnot wird so auch die Haushaltsführung zur voraussetzungsvollen „Gestaltungsaufgabe“ (Rerrich 1994, S. 203f.). Vor allem junge Erwachsene und Familien können Erwerbsarbeit und privates Alltagsleben nicht problemlos vereinbaren.

Damit stellt sich die Frage, ob die Haushaltsarbeit - trotz Abwesenheit einer Vollzeit-Hausfrau - weiterhin eine exklusiv private Aufgabe ist, oder ob sie teilweise und ergänzend auch als Erwerbsarbeit geleistet wird. Werden also stärker als bisher Dienstleistungen in Anspruch genommen? Die Modernisierung der Lebensformen würde einen steigenden Bedarf an Haushaltsdienstleistungen nahelegen. Jedoch ist trotz finanzieller Förderung durch steuerrechtliche Abschreibungsmöglichkeiten bislang kein regulärer Arbeitsmarkt für Haushaltsdienstleistungen entstanden.

Vor diesem Hintergrund lautete die Forschungshypothese der hier vorgestellten Studie, ${ }^{1}$ dass die Nachfrage nach Haushaltsdienstleistungen zwar nicht einkommensunabhängig ist, dass sie aber auch nicht in einer eindimensionalen Beziehung zum Einkommen von Haushalten bzw. zum Preis einer Dienstleistung steht. Als weitere - und wesentliche - Einflussfaktoren auf den realisierten Bedarf an Haushaltsdienstleistungen werden vielmehr Alltagspraktiken sowie sozio-kulturelle Einstellungen und Deutungsmuster vermutet.

Abschnitt 2 und 3 definieren zunächst den Untersuchungsgegenstand, das Erkenntnisinteresse und spezifizieren die Untersuchungshypothesen. Abschnitt 4 beschreibt die methodische Anlage des zugrunde liegenden Forschungsprojektes, bevor im nächsten Schritt zentrale Befunde des qualitativen Analyseteils (Abschnitt 5 und 6) wie auch der quantitativen Teilstudie (Abschnitt 7) präsentiert werden. Knappe Schlussfolgerungen werden in Abschnitt 8 gezogen.

\section{2 \\ Dienstleistungen für den privaten Haushalt}

Alltagssprachlich wird unter Haushaltsarbeit die Erledigung immer wieder anfallender Aufgaben verstanden wie Putzen, Kochen, Waschen, Aufräumen. In einer weiteren Perspektive geht es auch um die Organisation des Alltags und um die an Kommunikation gebundenen (z. B. die erzieherischen) Arbeiten. Im Anschluss an ethnografische Studien zur Haushaltsarbeit (Kaufmann 2005) wird im Folgenden ein solch weiterer Begriff unterstellt. Haushaltsarbeit wird als eine in soziale Deutungsmuster und in Vorstellungen vom „richtigen Leben“ eingebundene Tätigkeit

\footnotetext{
1 Es handelt sich um ein empirisches Forschungsprojekt, das unter dem Titel "Haushaltsdienstleistungen: Der potenziell Bedarf " von der Hans-BöcklerStiftung gefördert wurde (Projekt. 2005-783-3). Zur Untersuchungsanlage vgl. Abschnitt 4.
}

Birgit Geissler, Prof. Dr., lehrt Arbeitssoziologie an der Fakultät für Soziologie, Univer sität Bielefeld. Arbeitsschwerpunkte: Arbeitssoziologie, Professionalisierung von Dienstleistungsarbeit, Lebenslaufpolitik und Lebensplanung e-mail: birgit.geissler@uni-bielefeld.de 
verstanden. ${ }^{2}$ Auf der Grundlage dieses Konzepts werden die verbreiteten Vorbehalte gegen Haushaltsdienstleistungen analysiert.

Der Begriff der Haushaltsdienstleistungen richtet sich auf erwerbsförmig geleistete Dienste, die einen Bezug zum Haushalt haben - also Dienstleistungen für alltägliche Haushaltsarbeit, Kinderbetreuung und häusliche Alten- und Krankenpflege. ${ }^{3}$ Solche Dienste sind abgrenzbar von der öffentlichen Kinderbetreuung und von sozialen Diensten. Kommerzielle Dienstleistungen für Haushalte sind neuerdings in der Bundesrepublik eine schnell wachsende Branche. Ergänzend zu wohlfahrtsstaatlichen Diensten entstehen Angebote für die Betreuung von Klein- und Schulkindern, Sport- und Freizeitangebote, Essen-Bringdienste und anderes. Dieser Prozess wird begleitet von Debatten, die ein jahrelang aufgestautes Dienstleistungsdefizit beklagen und eine Ausweitung auch des privatwirtschaftlichen Angebots fordern. Dabei wird jedoch meist übersehen, dass Dienstleistungen, die im Haushalt selbst stattfinden, relativ wenig zu diesem Boom beitragen. Hier ist die Nachfrage nach wie vor gering. Dennoch haben auch diese Dienste an öffentlicher Aufmerksamkeit gewonnen. Arbeitsmarktpolitikerinnen und -politiker nehmen den Bedarf als sicher an und schließen auf eine entsprechende Nachfrage (Schupp et al. 2006). Die Politik identifiziert daher in den privaten Haushalten ein Arbeitsplatzreservoir. Zugleich sollen Erwerbsformen formalisiert und Arbeitsverhältnisse vertraglich geregelt werden, um die Schwarzarbeitsquote zu reduzieren.

Parallel zum familienpolitischen Diskurs über den Unterstützungsbedarf von Familien geht es in diesem arbeitspolitischen Diskurs um die Etablierung und Regulierung eines Arbeitsmarktes für gering qualifizierte Arbeitsuchende (vgl. Weinkopf 2003). Dafür sind verschiedene Modelle öffentlicher Förderung der Beschäftigung im Haushalt aufgelegt worden. Die geringe Wirksamkeit dieser Programme legt jedoch die Frage nahe: Bieten die Haushalte tatsächlich ein unausgeschöpftes Reservoir an Arbeitsplätzen? Zwar steigt die Nachfrage nach qualifizierten Diensten für Kinderbetreuung und Pflege - aber werden sie auch für die alltägliche Haushaltsarbeit in relevantem Maße nachgefragt? Mit anderen Worten: Artikuliert sich ein potenzieller Bedarf auch als Nachfrage auf dem Markt? Welche Haushalte nehmen
Haushaltsdienstleistungen in Anspruch und welche nicht? Welche Gründe für den Verzicht können identifiziert werden? Um diese Fragen geht es im Folgenden.

\section{3 \\ Analyseperspektive: Die Kultur der alltäglichen Lebensführung}

Bei den Initiativen zum „Arbeitsmarkt Haushalt" soll ein gegebenes Erwerbspotenzial aktiviert werden. Allerdings gibt es wenig gesichertes Wissen, inwieweit Haushaltsdienstleistungen tatsächlich in Anspruch genommen werden. Differenzierte Erkenntnisse über den potenziellen Bedarf und die Bedingungen einer Nachfrage für externe Dienste - ob von öffentlichen Trägern, von der Privatwirtschaft oder auch im „grauen Markt" erbracht - fehlen. Solches Wissen wäre jedoch erforderlich, um weitergehende rechtliche und sozialpolitische Fragen zu lösen und Subventionierungskonzepte zu gestalten. Es fehlen insbesondere Erkenntnisse dazu, in welcher Weise neben dem finanziellen auch andere Motive die Dienstleistungsnachfrage beeinflussen. Denn die private Form der alltäglichen Versorgung ist nicht nur gesellschaftlich institutionalisiert; sie ist vor allem auch in sozio-kulturellen Sinnbezügen verankert. Zu nennen sind hier soziale Leitbilder von Partnerschaft, Elternschaft und Kindheit, familiale Werte und alltagsweltliche Routinen und Lebensstile (Geissler 2003).

In der empirischen Studie, die der weiteren Argumentation zugrunde liegt (vgl. Fußnote 1), wurden daher sowohl die ökonomischen wie die kulturellen Entscheidungsgrundlagen der Dienstleistungsnachfrage erforscht. Ausgangsüberlegung war, dass die Herauslösung einer bisher regelmäßig im Haushalt verorteten Tätigkeit aus dem privaten Kontext, ihre Vergabe an externe Personen und die mit der Bezahlung verbundene Wertbestimmung dieser Tätigkeit nicht nur nach Kostengesichtspunkten entschieden wird. Sie muss in eine Neubestimmung der alltäglichen Lebensführung eingebettet und alltagskulturell legitimiert werden. Erst wenn die kulturellen Deutungsmuster dies „erlauben“, wird über die Umschichtung materieller Ressourcen nachgedacht, und erst dann werden Dienstleistungsangebote genutzt.
Diese These des kulturell motivierten Vorbehalts bezieht sich nicht auf individuelle Eigenheiten und hebt auch nicht auf Merkmale der jeweiligen Haushaltsform ab. Vielmehr rekurriert sie darauf, dass dem deutschen Wohlfahrtsregime eine implizite Strukturierung der Bereiche des Öffentlichen und des Privaten inhärent ist. Haushaltsbezogene Dienste - von der Tagesmutter bis zur Putzhilfe - tangieren diese Grenzen von Privatsphäre und Öffentlichkeit. ${ }^{4}$ Dies ist wörtlich zu nehmen: Die Erledigung von Haushaltsarbeit betrifft den privaten Raum im Sinne von Intimität und Vertrauensbeziehungen; das Bedürfnis nach Abschottung dieser Sphäre kann zur Ablehnung von Dienstleistungen führen.

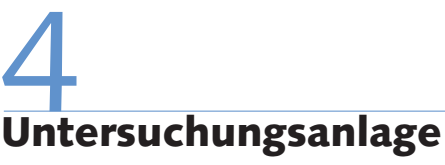

Die Forschungsarbeiten untergliedern sich in einen qualitativen und einen quantitativen Analyseteil.

(1) Die quantitative, repräsentative Erhebung fragt danach, wie die Belastung durch Haushaltsarbeit wahrgenommen wird und welche Dienstleistungen bereits in Anspruch genommen bzw. warum sie abgelehnt werden. ${ }^{5}$ Im Zentrum der qualitativen Teilstudie stehen soziale Praktiken, Alltagsroutinen und Deutungsmuster zur Haushaltsarbeit und entsprechende Erwartungen an bzw. Vorbehalte gegen Dienstleistungen. Im Einzelnen wurde untersucht

- wie die Belastung durch Haushaltsarbeit und Kinderbetreuung wahrgenommen wird;

- für welche Arbeitsfelder Dienstleistungen (nach Art, Trägerschaft, Preis etc.) in Anspruch bzw. nicht in Anspruch genommen werden;

2 Zu einer detaillierten Darstellung und Reflexion des wissenschaftlichen Forschungsstands in verschiedenen Disziplinen vgl. Geissler 2010.

3 Pflegedienstleistungen wurden aus forschungsökonomischen Gründen ausgeklammert. Für diesen Bereich ist zudem die Forschungslage besser als für Haushaltsdienstleistungen.

4 Vgl. dazu die Beiträge in Jurczyk/Oechsle 2008.

5 Die Erhebung erfolgte auf Basis eines standardisierten Fragebogens als Telefonbefragung in Haushalten mit mindestens einem Erwerbstätigen. $\mathrm{N}=$ 2019. Zu den Ergebnissen der repräsentativen Teilstudie vgl. Abschnitt 7. 


\begin{tabular}{|c|c|c|c|c|c|}
\hline \multicolumn{6}{|c|}{$\begin{array}{l}\text { Übersicht 1: Nutzer und Nichtnutzer von Haushaltsdienstleistungen - } \\
\text { Merkmale und Verteilung* }\end{array}$} \\
\hline & $\begin{array}{l}\text { Typ } 1 \\
\text { Pragmatische } \\
\text { Nutzer }\end{array}$ & $\begin{array}{l}\text { Typ } 2 \\
\text { Potenzielle } \\
\text { Nutzer }\end{array}$ & $\begin{array}{l}\text { Typ } 3 \\
\text { Interessierte }\end{array}$ & $\begin{array}{l}\text { Typ } 4 \\
\text { Passive }\end{array}$ & $\begin{array}{l}\text { Typ } 5 \\
\text { Ablehner }\end{array}$ \\
\hline Nutzung einer Dienstleistung & $\mathrm{Ja}$ & Nein & Nein & Nein & Nein \\
\hline Belastung durch Haushaltsarbeit & Ja/Nein & Ja & Nein & Nein & Ja/Nein \\
\hline Thematisierung einer Dienstleistung & Ja/Nein & $\mathrm{Ja}$ & Ja & Nein & Nein \\
\hline Zugeordnete Haushalte & 10 & 8 & 20 & 9 & 9 \\
\hline $\begin{array}{l}\text { *Typologie auf Basis der qualitativen Befunde. } \\
\text { Quelle: Darstellung der Autorin. }\end{array}$ & \multicolumn{5}{|c|}{ WSI mitTEILUNGEN } \\
\hline
\end{tabular}

- welche Motive für die Nutzung bzw. welche Vorbehalte es gegen Haushaltsdienstleistungen gibt;

- welche Ansprüche an die Qualität von Dienstleistungen und an die Formalqualifikation und die Kompetenzen der Arbeitskräfte gestellt werden;

- welche Relevanz die sozialrechtliche Absicherung von Haushaltsdienstleisterinnen und -dienstleistern hat.

(2) In der qualitativen Teilstudie wurden 56 thematisch strukturierte Interviews in Bielefeld und Leipzig durchgeführt. Den größten Anteil machten die Interviews mit Familien ${ }^{6}$ aus (etwa $40 \%$ ). Ferner wurden Alleinerziehende als eine Familienform mit besonderen Merkmalen, Paarhaushalte ohne Kinder, Alleinlebende und (wenige) Wohngemeinschaften befragt. Die durchschnittliche Haushaltsgröße des Samples beträgt 2,5 Personen (im Bundesdurchschnitt 2,1 Personen). Die Altersstruktur der Befragten (drei Viertel im Alter zwischen 30 und 45 Jahren) ist aufgrund des Auswahlkriteriums, dass mindestens ein Erwerbstätiger im Haushalt leben sollte, viel niedriger als im Bundesdurchschnitt. Erwähnenswert ist schließlich noch die untypische Einkommensverteilung. ${ }^{7}$ Das relativ hohe Durchschnittseinkommen im Sample ist darauf zurückzuführen, dass nur Haushalte mit mindestens einem Erwerbstätigen befragt wurden. In einem Großteil der Mehrpersonenhaushalte sind zudem zwei Personen in Vollzeit erwerbstätig (25 von 37). In gut der Hälfte der befragten Haushalte (29 von 56) leben Kinder. Die heuristische Annahme, dass moderne Lebensformen und Einstellungen eher im städtischen Kontext anzutreffen sind, wird unter anderem dadurch bestätigt, dass sich im Sample kein Haushalt mit einer „klassischen“ Hausfrau befindet, obwohl dies kein Auswahlkriterium war. Unter den befragten Haushalten befinden sich zehn, die aktuell und dauerhaft als Arbeitgeber für Haushaltsdienstleistungen auftreten, und vier weitere, die dies in der Vergangenheit getan haben.

Die Interviews gliederten sich in die Themenkomplexe (1) Alltag und Lebensführung, (2) eigene Haushaltsarbeit, (3) Haushaltsdienstleistungen und (4) Kinderbetreuung. Bei den Fragen zu Lebensführung und Haushaltsarbeit (1 und 2) interessierten zum einen die Standards der Haushaltsführung, zum anderen das Belastungsempfinden. ${ }^{8}$ Im Interviewteil zu Dienstleistungen (3) wurden die Hintergründe für einen (gegebenenfalls ungedeckten) Bedarf sowie die Motive für die Nutzung oder Nichtnutzung erfragt. Im vierten Themenkomplex zeigte sich, dass es wohl zu kaum einem anderen Thema so viele unterschiedliche Einschätzungen gibt wie zu der Frage, was für Kinder gut ist - mit Ausnahme der durchweg positiven Einstellung zum Kindergarten als institutioneller Form der Kinderbetreuung.

Zusammenfassend ist festzuhalten, dass die Thematik viel komplexer ist als vorausgesehen: Die verschiedenen Haushaltsformen sind in sich noch stark differenziert, sodass keine Aussagen über die Singles oder die Familien zu treffen sind. Diese Differenzierung drückt sich unter anderem auch in den verschiedenen Erledigungstypen von Haushaltsarbeit aus, in den unterschiedlichen Sauberkeits- und Ordnungsstandards, im Belastungsempfinden und in der Frage, ob Haushaltsarbeit als Arbeit anzusehen ist. Es wird deutlich, dass sich empirisch keine Muster entlang verschiedener Haushaltsformen aufzeigen lassen.

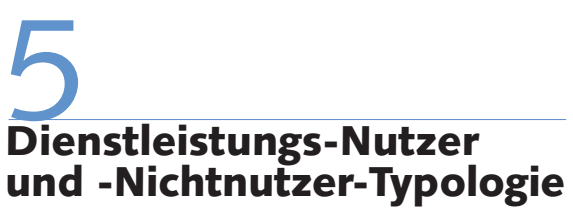

Welche Bedeutung hat die spezifisch deutsche sozio-kulturelle Tradition der Über- höhung des Privaten und der Abschottung der Familie vom öffentlichen Raum? Behindert auch bei der jüngeren, städtischen Bevölkerung die „Schwelle des Privaten“ die Nachfrage nach Haushaltsdiensten, oder sind die entsprechenden Deutungen im Wandel begriffen? Welche Widerstände ergeben sich aus der Eigenlogik des Privaten? Die Interviews brachten eine Fülle von Informationen zu diesen Fragen. Im Folgenden werden wesentliche Ergebnisse in typologischer Zuspitzung dargestellt (Übersicht 1). Die Typen wurden entlang der Ähnlichkeiten in den Praktiken der Lebensführung sowie in den Einstellungen hinsichtlich Haushaltsarbeit und Dienstleistungen konstruiert. Es handelt sich hier um das Verhältnis zu Haushaltsdienstleistungen im engeren Sinne, d.h. ohne Kinderbetreuung.

Die erste Unterscheidung der Typen bezieht sich auf die aktuelle Inanspruchnahme bzw. Nicht-Inanspruchnahme von Dienstleistungen. Neben dem „Pragmatischen Nutzer" (Typ 1) wurden vier weitere Typen identifiziert, die aus unterschiedlichen Gründen keine Haushaltsdienste in Anspruch nehmen (Typ 2-5). ${ }^{9}$ Bei den Gründen für den Verzicht sind drei Gruppen auszumachen: Zum einen gibt es Motive, die sich aus der finanziellen Lage und anderen Elementen der aktuellen Lebensführung ergeben, also nicht auf Leitbilder und Deutungen zurückgehen. Zum anderen spielen politisch motivierte Einstellungen und schließlich - wie angenommen kulturell fundierte Vorbehalte eine Rolle.

6 Die Lebensform Familie wurde hier definiert als „verheiratete Erwachsene, die mit Kindern zusammen leben“. Der umfassendere Familienbegriff des Statistischen Bundesamt (2006, S. 27) wurde nicht übernommen, um der besonderen Lebenssituation Alleinerziehender Rechnung zu tragen.

7 Insgesamt 27 der 56 interviewten Haushalte verfügen über ein Netto-Monatseinkommen von über $2.500 €$ und nur 13 liegen unter $1.500 €$ (hierunter fallen fünf der acht Alleinerziehenden).

8 Die Auswertung ergab Unterschiede in den Ansprüchen an Sauberkeit und Ordnung sowie drei Arten der Erledigung der eigenen Haushaltsarbeit: 1. eine spontane, unregelmäßige Erledigung, 2. eine regelmäßige, routinisierte Erledigung, 3. eine planmäßige und strukturierte Erledigung. Auch die Wertschätzung der Haushaltsarbeit war unterschiedlich (distanziert-abwertende Haltung, gleichgültige Haltung, positive Wertschätzung).

9 Typen-bildend waren zentrale Codes, die häufig im Material vorkamen und die besonders trennscharf wirkten. Dabei werden inhaltliche Sinnzusammenhänge kenntlich und Verknüpfungen zwischen Lebenslage, Lebensführung und Deutungsebene hergestellt. 
In den Typen 2 und 3 sind die Argumente zentral, die als „Lebensführungs-Vorbehalte" bezeichnet werden können. Bei den Typen 4 und 5 sind kulturell oder politisch fundierte Vorbehalte und Ablehnungsgründe vorherrschend.

Interessanterweise lassen sich die befragten Haushalte auch nach zwei weiteren diskriminierenden Merkmalen typisieren. Neben der Nutzung selbst erwiesen sich die Fragen, ob sich die Haushaltsmitglieder durch die Arbeit im Haushalt belastet fühlen und ob über eine Dienstleistungsnutzung bereits einmal gesprochen wurde, als trennscharf. Folgende (Nicht-)Nutzertypen sind zu unterscheiden:

Die Haushalte, die als „Pragmatische Nutzer" bezeichnet werden (Typ 1), sind die Referenzgruppe für die Bestimmung der anderen Typen. Hier kommt es nur auf die Tatsache der aktuellen Inanspruchnahme einer Dienstleistung an; die anderen beiden Merkmale treffen nicht durchweg zu. Die „Potenziellen Nutzer“ (Typ 2) fühlen sich durch Haushaltsarbeit belastet und haben bereits schon mal darüber nachgedacht, Dienstleistungen in Anspruch zu nehmen. Der Umsetzung dieser Idee steht in erster Linie die Einkommenssituation im Wege. Die „Interessierten“ (Typ 3) fühlen sich dagegen durch die Arbeit im Haus nicht besonders belastet, haben aber dennoch eine Inanspruchnahme von Haushaltsdienstleistungen bereits einmal erwogen. Interessante Unterschiede zeigten sich bei denjenigen Haushalten, die keine Dienstleistung nutzen und dies auch nicht thematisiert haben; sie wurden daher zwei Typen zugeordnet. Während bei den „Passiven“" (Typ 4) wegen der alltäglichen Routine des "Selbermachens" und kultureller Vorbehalte Dienstleistungen gar nicht in den Blick kommen, stehen bei den „Ablehnern" (Typ 6) politische und kulturelle Vorbehalte sowie ein generelles Misstrauen gegenüber den Anbietern von Haushaltsdienstleistungen im Vordergrund.

\subsection{NUTZUNG VON HAUSHALTSDIENSTLEISTUNGEN - PRO UND KONTRA}

(Typ 1): Die „Pragmatischen Dienstleistungsnutzer": Sie artikulieren und realisieren ihren Bedarf an Unterstützung bei der Haushaltsarbeit (in der Regel eine Putzhilfe), um Zeit zu gewinnen oder sich den Alltag zu erleichtern. Das Motiv einer manifesten Überforderung spielt jedoch eine untergeordnete Rolle. Die tägliche Arbeit wäre zu bewältigen, man nutzt die Dienstleistungen hauptsächlich, um mehr Freiräume für sich oder die Familie zu haben. Es handelt sich bei den zehn Haushalten dieses Typus' um vier Familienhaushalte und eine Alleinerziehende sowie drei SingleHaushalte und zwei Paarhaushalte. Die Kinder sind alle jünger als zwölf Jahre und brauchen viel Betreuung. In den Haushalten diesen Typus' sind die Erwachsenen vollzeit-erwerbstätig, und sie sind mehrheitlich höheren Einkommensgruppen zuzurechnen (über $2.500 €$ monatliches HaushaltsNetto-Einkommen). Vorbehalte gegenüber Dienstleistungen werden kaum genannt. Der Schutz der Privatsphäre spielt eine eher geringe Rolle und die Dienstleistenden werden als Partner im Sinne einer Geschäftsbeziehung betrachtet und behandelt.

(Typ 2): Die „Potenziellen Nutzer“: Diese Gruppe zeichnet sich durch einen objektiv gegebenen und auch artikulierten Bedarf an Unterstützung aus, der jedoch ungedeckt bleibt. Es wird davon ausgegangen, dass Haushaltsdienste zu teuer seien; eine charakteristische Aussage dazu lautet: „Geld. Es ist definitiv nur das Geld.“ Das finanzielle Argument ist nicht kulturell-ideologisch unterfüttert, also kein Vorwand für andere Vorbehalte, die auch kaum geäußert werden. Haushaltsdienstleistungen in Anspruch zu nehmen, erscheint wünschenswert und vorstellbar; die Haushalte suchen Unterstützung, um entlastet zu werden und Zeit zu gewinnen. Gründe für den Verzicht liegen hier also in der Lebensführung, konkret im verfügbaren Geld. Auch hier ist es überwiegend das unliebsame Putzen, dessen man sich gern entledigen würde.

Diesem Typ sind drei Familien- und vier Alleinerziehenden-Haushalte zuzuordnen. Die Befragten dieser Gruppe sind alle jünger als 50 Jahre. Und die Haushaltseinkommen liegen zwischen $1.000 €$ und $3.500 €$. Im Vergleich mit den anderen Typen sind hier die eher großen Haushalte vertreten. Die Potenziellen Nutzer erledigen die Haushaltsarbeit überwiegend strukturiert und planvoll, obwohl sie einen deutlichen Widerwillen dagegen äußern und den (belastenden) Arbeitscharakter betonen. Die Aussagen deuten auf Stress durch die Alltagsorganisation, das Leben mit Kindern und die Erwerbsarbeit hin. Diese Aussagen, verbunden mit dem häufig beklagten Zeitmangel, lassen auf einen objektiven Bedarf - der nur aus finanziellen Gründen nicht gedeckt wird - schließen. In diesen Haushalten wird die Relevanz des Einkommens offen kommuniziert, während dies in den nachfolgenden Typen seltener der Fall ist bzw. das finanzielle Argument mit anderen Vorbehalten einhergeht.

(Typ 3): Die „Interessierten“: Sie bekunden auf der einen Seite ein Interesse an Dienstleistungen, haben aber gleichzeitig kulturell begründete Vorbehalte. $\mathrm{Zu}$ diesem Typus gehören zwölf Familien, die überwiegend jüngere Kinder haben. Auffallend ist, dass sich keine Alleinerziehenden in dieser Gruppe befinden. Die Altersstruktur ist heterogen; ebenso die Einkommensstruktur. In dieser Gruppe finden sich die meisten Haushalte mit einer traditionellen Rollenverteilung. Die Haushalte dieses Typus' unterscheiden sich stark hinsichtlich der Zeitstruktur. Es gibt sowohl Haushalte mit einem durchorganisierten als auch solche mit einem turbulenten oder mit einem komplett routinisierten Alltag. Anders als bei den pragmatischen und den potenziellen Nutzern ist Haushaltsarbeit hier nicht durchgängig negativ besetzt. Neben dem Widerwillen finden sich auch gleichgültige („es muss eben gemacht werden") und positive Einstellungen. Als Gründe für den Verzicht auf Haushaltsdienstleistungen werden vor allem Gewohnheiten und die Präferenz des „Selbermachens" genannt. Außerdem spielen der Schutz der Privatsphäre und ein befürchteter Mehraufwand eine Rolle. Dennoch sind diese Vorbehalte nicht so groß, als dass sich die Befragten nicht vorstellen könnten, Haushaltsdienstleistungen $\mathrm{zu}$ nutzen quasi als möglichen „Luxus“.

(Typ 4): Die „passiven“ Haushalte: Diese Haushalte haben kein Interesse an Haushaltsdienstleistungen. Nur wenige der ganz jungen Haushalte gehören zu diesem Typ; sechs der neun typenbildenden Haushalte sind der Gruppe der über 40-Jährigen zuzurechnen. In der Hälfte der Haushalte leben Kinder. Ihre Lebensführung zeichnet sich durch Konstanz und Routine aus. Weder die Alltagsorganisation noch die Haushaltsarbeit werden als belastend wahrgenommen. Haushaltsarbeit erfährt hier die höchste Wertschätzung unter allen Interviewten. Sie wird vergleichsweise regelmäßig und mit viel Sorgfalt erledigt und nicht als Arbeit im negativen Sinn definiert. Man hat hohe Ansprüche an Sauberkeit und Ordnung.

Anders als die Haushalte der ersten drei Typen haben die Passiven eine negative 
Einstellung zu Haushaltsdienstleistungen. Zum einen, weil man die Arbeit gut selbst erledigen kann. Zum anderen werden Vorbehalte, zum Teil auch Misstrauen gegen Fremde im Haushalt geäußert, die die Privatsphäre gefährden könnten.

(Typ 5): „Ablehner“: Von den Befragten dieses Typus' werden Haushaltsdienstleistungen - ungeachtet eines gegebenen Bedarfs - dezidiert und mit Überzeugung abgelehnt. Überwiegend sind hier Haushalte ohne Kinder anzutreffen. Ihr Einkommen variiert breit. Die meisten Befragten haben einen geordneten Alltag mit viel Routine. Das Verhältnis zur Haushaltsarbeit ist sehr heterogen. Gleiches gilt für die Art und Weise, wie Haushaltsarbeit erledigt wird. Doch selbst diejenigen, die explizit einen Widerwillen gegen Haushaltsarbeit haben, lehnen es strikt ab, Dienstleistungen in Anspruch zu nehmen. Sie wollen - ähnlich wie die Passiven - keine „Fremden“ im Haushalt haben bzw. haben ein grundsätzliches Misstrauen gegenüber Dienstleistern. Eine Untergruppe in diesem Typus artikuliert zudem eine diffus politisch motivierte Kritik daran, dass es Haushaltsdienstleistungen überhaupt gibt. Da ein solches Arbeitsangebot nur „aus sozialer Not“ entstehen könne, möchte man dies nicht ausnutzen; es wäre unangenehm, oder man hätte als Arbeitgeber ein „schlechtes Gewissen“. Diese Interviewten wollen sich also von „Dienstboten-Ausbeutern" abgrenzen und lehnen Haushaltsdienste pauschal ab.

\subsection{ZWISCHENFAZIT}

Insgesamt drei Typen (die „Interessierten“, „Potenziellen Nutzer“ und „Pragmatischen Nutzer") - und damit die Mehrheit unseres Samples - zeichnen sich durch eine mehr oder weniger stark ausgeprägte Nachfragebereitschaft nach Haushaltsdienstleistungen aus. Vorbehalte signalisieren die „Passiven“ sowie die „Ablehner“. Vor allem aber verdeutlicht die Typologie, dass die Lebenslage den objektiven Bedarf nach Haushaltsdienstleistungen (im engeren Sinne) stark beeinflusst. Vor allem Kinder im Haushalt sowie Vollzeiterwerbstätigkeit erhöhen den Bedarf, allerdings wirkt sich das nicht eindeutig auf das Nachfrageverhalten aus. Auch zeigt sich ein Zusammenhang zwischen Einkommenshöhe und Dienstleistungsnachfrage. Allerdings: Die Haushalte des Typs „potenzieller Nutzer" haben mehrheitlich ein relativ geringes Einkommen.
Doch jenseits dieser „harten“ Faktoren sind es eindeutig auch Aspekte der Lebensführung - wie die Wertschätzung von disponibler Zeit - sowie Einstellungen wie das Bedürfnis nach geschützter Privatheit, die die Nachfrage nach oder Ablehnung von Haushaltsdienstleistungen bestimmen. Letztlich können Aspekte des Lebensstils sogar ein größeres Gewicht als ökonomische Faktoren haben: So sind es im Fall der „pragmatischen“ und „potenziellen“ Nutzer eher Familien, die sich selbst und ihren Kindern, bei zum Teil ausgeprägtem beruflichem Engagement beider Elternteile, mehr Freizeit und Familienzeit verschaffen wollen und deswegen einen Bedarf an Dienstleistungen nutzen oder erwägen.

Von Bedeutung ist offenbar auch das Alter: Bei den älteren Befragten sind eher restriktive Einstellungen anzutreffen, die einer Delegation von Haushaltsarbeiten entgegenstehen. Hier dominieren vor allem traditionelle häusliche Werte, ein Ethos des "Selbermachens" sowie die Angst, eine „Fremde“ im Haus könnte die Privatsphäre verletzen. Bemerkenswert ist, dass wir solche Einstellungen und Deutungsmuster bei einer größeren Anzahl von Haushalten gefunden haben, obwohl die traditionelle Lebensform der Hausfrauenehe, in der die Haushaltsarbeit die Domäne - und der Stolz - der Hausfrau ist, im Untersuchungssample nicht vertreten ist.

Die Nicht-Nutzertypen (Typ 4 und 5) bestätigen klar die forschungsleitende These, dass die Inanspruchnahme von Dienstleistungen zwar nicht einkommensunabhängig, jedoch stark von sozio-kulturellen Einstellungen und Deutungsmustern bestimmt ist. Der hohe Altersdurchschnitt bei diesen Typen bestätigt zudem die Annahme, dass solche Deutungsmuster, die der Inanspruchnahme von Haushaltsdienstleistungen entgegenstehen, auf dem Rückzug sind. Vor allem jüngere Menschen sind aufgrund veränderter Lebensformen und Einstellungen offener für Dienstleistungen.

\section{Kinderbezogene Dienstleistungen}

Die aktuellen öffentlichen Diskurse zum Kindeswohl und zur besten Betreuung kleiner Kinder reflektieren die bereits stattgefundene wie stattfindende Modernisierung der Lebensformen. Sie sind zugleich von der positiven Einstellung zu früher Fremdbetreuung beeinflusst, die in den neuen Bundesländern weiterhin vorherrscht. Keineswegs mehr so eindeutig wie noch in den 1980er Jahren dominiert heute die Meinung, Kinder „gehörten zur Mutter“. Eine derart traditionelle Sichtweise war in unserer Studie der Ausnahmefall. Die Befragten aus Haushalten mit Kindern (aber auch Kinderlose) sind sich darin einig, dass Kinder nicht ausschließlich zu Hause betreut werden müssen und dass eine externe Betreuung legitim und sinnvoll ist. Auch die Betreuung nach dem Unterricht (Ganztagsschule oder Hort) ist in den befragten Haushalten mit Kindern sehr verbreitet und akzeptiert.

Da in Ost- wie Westdeutschland die überwiegende Mehrheit der Drei- bis Sechsjährigen einen Kindergarten besucht, es hierzu also keinen dringenden Forschungsbedarf gibt, haben wir nach dem Betreuungsbedarf für jüngere Kinder gefragt: zum einen nach der Nutzung nichtinstitutioneller Betreuungsformen (Kinderfrau, au-pair, Krabbelgruppe, Tagesmutter), zum anderen nach Kinderhort und Kinderkrippe. Stärker als beim Kindergarten nutzen Eltern diese Betreuungsformen, um (vor allem) der Mutter den frühen beruflichen Wiedereinstieg zu ermöglichen. Diese Betreuungsformen sind daher eher als der Kindergarten als kinderbezogene Dienstleistungen zu verstehen. Dabei werden besonders in Ostdeutschland Kinderbetreuungseinrichtungen den sogenannten familiennahen Betreuungsformen vorgezogen. So wurden in Leipzig nicht-institutionelle Formen der Kleinkindbetreuung (in erster Linie die Tagesmutter) lediglich als Ersatz angesehen, wenn kein Krippenplatz zur Verfügung steht.

Die Nutzungsformen dieser Dienstleistungen sind nicht so breit gefächert wie die von Haushaltsdienstleistungen im engeren Sinn. Alle Haushalte mit Kindern nutzen bzw. nutzten Betreuungsdienste. Unterschiedlich war lediglich der explizit oder implizit artikulierte Bedarf nach weiteren Dienstleistungen zur Kinderbetreuung. Im Ergebnis bilden sich nur zwei Typen heraus.

(1) Typ A: Nutzer (und ehemalige Nutzer) von kinderbezogenen Dienstleistungen: Beim Typ A ist der aktuelle (oder wenn die Kinder mittlerweile älter sind: ehemalige) Betreuungsbedarf recht weitgehend ge- 
deckt. Die Haushalte dieses Typs fühlen sich nicht übermäßig belastet, auch weil sie neben einer institutionalisierten Form der Kinderbetreuung ein festes Netzwerk aus Familie und/oder Freunden und Bekannten haben. Die Bedeutung dieses Netzwerkes wird immer wieder betont, weil es als Ergänzung zu den institutionellen Lösungen gerade in den Tages-Randzeiten und in Notsituationen eine große Rolle spielt. Informelle Netzwerke scheinen eine Flexibilität zu ermöglichen, die mit Dienstleistern allein nicht oder nur zu sehr hohen Preisen erreicht werden kann. Entgegen der Erwartung befinden sich in diesem Typ genau so viele Alleinerziehende wie im Typ B.

Die Aufgeschlossenheit gegenüber kinderbezogenen Dienstleistungen zeigt sich auch im Verhältnis zu anderen Dienstleistungen. Fast alle Haushalte dieser Gruppe sind bei der Typologie der Haushaltsdienstleistungen im engeren Sinne den Typen eins bis drei zuzuordnen. Das heißt, diese Gruppe steht Dienstleistungen generell offen gegenüber.

(2) Typ B: Nutzer von kinderbezogenen Dienstleistungen mit weitergehendem Bedarf: Auch die Haushalte des Typs B nutzen kinderbezogene Dienstleistungen. Jedoch wünschen sie sich entweder weitere Hilfe oder eine Ausweitung des derzeitigen Angebots. Das meint: zusätzliche Betreuungsmöglichkeiten in Randzeiten, also Betreuung am Wochenende, Flexibilisierung der Öffnungszeiten oder Angebote für stundenweise Betreuung. Auch der Wunsch nach Fahrdiensten zu den Kindereinrichtungen ist virulent. $\mathrm{Zu}$ diesem Typ zählen alle Befragten, die auf kein informelles Netzwerk zurückgreifen können. Wie bei Typ A sind die Einstellungen zur Kinderbetreuung nicht unabhängig von denen zu Haushaltsdienstleistungen. Auch diese Haushalte lassen sich zum Großteil den Typen 1 bis 3 zuordnen, stehen Dienstleistungen also weitgehend positiv gegenüber.

Anders als bei den fünf Typen von (Nicht-)Nutzern von Haushaltsdienstleistungen im engeren Sinne bieten Lebenslagen und Lebensführung der Haushalte, die den Typen A und B zugeordnet sind, ein heterogenes Bild. Das gilt sowohl für das Alter (von Eltern wie Kindern), für die Zahl der Kinder wie auch für die Haushaltsgröße und das Einkommen. Selbst die Strukturiertheit der Haushaltsarbeit, die Vergabe von Haushaltsdienstleistungen im engeren Sinne und die Arbeitsteilung im Haushalt, sind heterogen. Beide Typen sind
- ohne eindeutige Schwerpunktsetzung in Ost- wie in Westdeutschland anzutreffen.

Die Berufstätigkeit von Frauen ist für alle Befragten eine Selbstverständlichkeit. In keinem Fall wird ein Widerspruch zwischen der Berufstätigkeit und dem Anspruch, eine gute Mutter zu sein, gesehen. Das Deutungsmuster, dass ein Kind nur bei der Mutter gut aufgehoben sei, findet keine Unterstützung. ${ }^{10}$ Es wird abgelöst von der Einstellung, dass es weniger auf die Quantität als auf die Qualität der Zeit mit dem Kind ankommt und spitzt sich sogar soweit $\mathrm{zu}$, dass einige Befragte eine Berufstätigkeit der Mutter als Voraussetzung für ein gutes Verhältnis zum Kind sehen. Ein prägnantes Zitat: „Also ich denke mal, je berufstätiger man ist, umso besser ist man als Mutter. (...) Also ich bin definitiv eine gute Mutter, wenn ich Ziele habe oder wenn ich Aufgaben habe, die anerkannt werden." (Int. 55). Viele Befragte betonen, dass sie die gemeinsame Zeit mit dem Kind am Abend oder am Wochenende dafür bewusst gestalten; sie sehen die externe Kinderbetreuung also nicht als Konkurrenz zur eigenen Fürsorge und Verantwortung.

\section{1 Gründe für die Ablehnung von Haushaltsdienstleis- tungen}

Ergänzend zu unserer qualitativen Analyse haben wir auch im quantitativen Analyseschritt den potenziellen Bedarf nach allgemeinen und nach kinderbezogenen Dienstleistungen erfragt ${ }^{11}$ sowie die Gründe, die ihrer Inanspruchnahme entgegenstehen. Darüber hinaus ging es um die Qualitätserwartungen. Und schließlich haben wir angesichts des Informationsdefizits $\mathrm{zu}$ Schwarzarbeit im Haushalt - auch die Einstellung zur Schwarzarbeit und (bei den Dienstleistungsnutzern) die Entlohnungsformen erhoben. Explizit nicht Gegenstand der repräsentativen Erhebung war die „Preis-Frage“, also welche Lohnhöhe für Dienstleistungen die Haushalte als angemessen ansehen und selber bereit sind, $\mathrm{zu}$ zahlen. Letzteres war bereits Gegenstand der methodisch verwandten GIB-Studie von 2006 (vgl. GIB 2007).

Die Ergebnisse der quantitativen Teilstudie sind für deutsche Haushalte mit mindestens einem Erwerbstätigen reprä- sentativ. Nicht unerwartet ist der Befund, dass die überwiegende Mehrheit (91\%) der Haushalte keine Haushaltsdienstleistungen in Anspruch nimmt - trotz niedriger Preise auf dem informellen Markt.

Das finanzielle Argument spielt für den Verzicht ohnehin eine vergleichsweise untergeordnete Rolle. Die meisten Befragten lehnen Haushaltsdienste ab, weil sie diese Arbeit „schon immer selber gemacht" haben (92\%), weil sie denken, dass man diese Arbeiten selber erledigen sollte (75\%) oder weil sie die Haushaltsarbeit gerne machen $(65 \%)$. Erst an vierter Stelle werden finanzielle Gründe genannt (62\%). ${ }^{12} \mathrm{Da}$ mit wird auch in der repräsentativen Umfrage die Annahme bestätigt, dass die Inanspruchnahme haushaltsbezogener Dienstleistungen nicht nur vom Einkommen, sondern auch in hohem Maße von Alltagsroutinen und von Einstellungen und Deutungsmustern abhängig ist.

Die Einstellung zur Schwarzarbeit ist überwiegend negativ. Auf die Frage „Was halten Sie von folgender Aussage: Dass Haushaltshilfen schwarzarbeiten, ist völlig normal“ äußerte nur ein Viertel aller Befragten Zustimmung („stimme völlig zu“ und „stimme zu“). Ablehnend dagegen verhielten sich $44 \%$ der interviewten Personen („stimme gar nicht zu“ und „stimme nicht zu"). Weitere $28 \%$ hatten keine zustimmende oder ablehnende Meinung („teils/teils“). Die eigene Praxis sieht allerdings etwas anders aus. Von den Haushalten, die eine bezahlte Putzhilfe beschäftigen, geben $45 \%$ an, die Bezahlung dieser Dienstleistung gegen Rechnung oder über

10 Die Mehrheit der Befragten spricht sich jedoch dafür aus, dass eine Mutter in den ersten ein bis zwei Lebensjahren des Kindes "zu Hause bleiben“" soll.

11 Als Haushaltsarbeit wurden folgende Tätigkeiten in der Befragung definiert: Putzen, Wäsche, Einkaufen, Kochen und Kinderbetreuung. Zu berücksichtigen ist, dass Haushaltsarbeit in Haushalten mit Kindern weitere kommunikative Dimensionen bekommt. In den Fragenformulierungen wurde deswegen nach dem Alter der Kinder im Haushalt (bis 8 Jahre und 9-14) unterschieden.

12 Um die Ablehnungsmotive zu erfragen, haben wir neun Gründe zur Auswahl gestellt, von denen mehrere genannt werden durften. Im Anschluss an die aus den qualitativen Interviews gewonnenen Erkenntnisse wurden sowohl alltagskulturell fundierte Einstellungen wie auch sich aus der Lebensführung ergebende "praktische" Verzichtsgründe vorgegeben. Um eine Beeinflussung des Antwortverhaltens durch die Abfolge der Vorgaben auszuschließen, wurden diese in zufällig wechselnder Reihenfolge, also randomisiert, abgefragt. 
einen Arbeitsvertrag geregelt zu haben. Weitere $23 \%$ erledigen die Abrechnung „ohne Belege“, und noch einmal $23 \%$ bezahlen "mal so, mal so“ (keine Angabe: $9 \%$.) Es kann also davon ausgegangen werden, dass etwa die Hälfte der ArbeitgeberHaushalte „schwarz“ arbeiten lässt, während die andere Hälfte die Haushaltsdienstleistungen legal bezahlt. Dieser Befund weicht zumindest erheblich nach unten von den verbreiteten und höheren Schätzungen zur Schwarzarbeit bei haushaltsbezogenen Dienstleistungen ab.

\section{Schlussfolgerungen}

Welche Schlüsse können aus den Ergebnissen der Studie gezogen werden? Kulturell motivierte Vorbehalte gegen haushaltsbezogene Dienstleistungen sind in zahlreichen Haushalten anzutreffen. Sie sind offenbar langfristig handlungsleitend. $\mathrm{Zu}-$ gleich ist die soziale Zusammensetzung der Haushalte, die diese Vorbehalte artikulieren, interessant: Es sind jene mit höherem Altersdurchschnitt und solche, in denen keine Kinder (mehr) leben. Die Annahme ist also nicht unplausibel, dass das Bedürfnis nach einer rigiden Abgrenzung des privaten Bereichs in der jüngeren Generation schwächer ausgeprägt ist als bei den Älteren.

Um die weitere Entwicklung von Haushaltsdienstleistungen als Erwerbssektor einzuschätzen, sind besonders die beiden Haushaltstypen unseres Samples interessant, die lediglich aus ihrer aktuellen Lebenslage und Lebensführung heraus keine Dienstleistungen in Anspruch nehmen, jedoch keineswegs eine grundsätzliche Abwehr gegenüber Dienstleistern im Haushalt haben. Gerade mit Blick auf diese Gruppe stellt sich die Frage, ob sich aus dem ungedeckten Bedarf, der bei einem beträchtlichen Teil dieser „Nicht-Nutzer“ gegeben ist, eine Nachfrage entwickeln kann - vor allem eine Nachfrage, die in regulärer Form (und nicht als Schwarzarbeit) realisiert wird. Prognosen sollen hierzu nicht gegeben werden, jedoch einige abschlie-
Bende Überlegungen, die grundlegende Voraussetzungen für das potenzielle Wachstum von Haushaltsdienstleistungen skizzieren:

Private Haushaltsarbeit zeichnet sich durch komplexe, an den Bedürfnissen der Haushaltsmitglieder orientierte Arbeitsformen aus, die habituelle und emotionalinteraktive Komponenten haben (Geissler 2006). Von der Seite potenzieller Nachfrager werden Dienstleistungen explizit oder implizit an diesen eigenen, meist unausgesprochenen Praktiken und Standards für Haushaltsarbeit gemessen. Die im Vergleich mit anderen Dienstleistungen geringe Inanspruchnahme auch in den Haushalten, die keine rigide Abschottung ihres Privatbereichs für notwendig halten, hat daher auch damit zu tun, dass diese Praktiken und Standards der eigenen Arbeit normativ verankert sind und zugleich als Elemente des Habitus' weitgehend vorbewusst bleiben. Daher ist der Umfang möglicher Nachfrage von der Sache her begrenzt. Es ist nicht davon auszugehen, dass private Haushaltsarbeit vollständig durch Dienstleistungen ersetzt werden kann. Dennoch ist der Anteil von etwa $10 \%$ aller Erwerbstätigen-Haushalte in Deutschland, die Dienstleistungen (zum Teil in geringem Umfang) tatsächlich nutzen, sehr niedrig.

Diese Überlegungen führen zur Annahme, dass zwei Bedingungen gegeben sein müssen, damit sowohl die „Potenziellen Nutzer", die ja explizit ihren Bedarf an Haushaltsdienstleistungen artikulieren, als auch die „Interessierten“ ihre von der alltäglichen Lebensführung her bestimmten Vorbehalte relativieren (und dann auch gegebenenfalls finanzielle Ressourcen umschichten). Die eine Bedingung betrifft die Angebotsseite: Eine Haushaltsdienstleistung müsste so gestaltet sein und ausgeführt werden, dass sie mit dem Habitus und den spezifischen Vorstellungen des Arbeitgeberhaushalts nicht grundsätzlich in Widerspruch gerät. Das gilt sowohl für routinisierte Arbeitsvollzüge wie auch für interaktiv geprägte Tätigkeiten, etwa in der Kinderbetreuung.

Das aber stellt spezifische Anforderungen an die Beobachtungsgabe, Flexibilität und Anpassungsbereitschaft der Dienstleisterinnen und Dienstleister. Und solche Erwartungen sind wahrscheinlich leichter von Personen zu erfüllen, die für diese Arbeit ausgebildet sind und deren Arbeitssituation und Arbeitsverhältnis rechtlichen und sozialen Mindeststandards genügen. Davon ist die Lage in Deutschland jedoch weit entfernt. Bei den Haushaltsdienstleistungen handelt es sich aktuell um einen weitgehend unregulierten Erwerbssektor, außerhalb organisationaler Einbindung, mit uneinheitlichen Vorgaben für die Arbeitsausführung und die Qualität der Dienstleistungen. Mit Ausnahme der öffentlichen Kinderbetreuung gibt es in diesem Sektor weder ein professionelles Selbstverständnis noch gültige Standards für Entlohnung und Arbeitsbedingungen.

Die andere Bedingung betrifft die auftraggebenden Haushalte. Hier bieten die Ergebnisse zur Motivlage und zum Habitus der Nutzer-Haushalte (Typ 1) relevante Hinweise: Die Haushalte, die Dienstleistungen in Anspruch nehmen, geben die als besonders belastend empfundenen Tätigkeiten nicht nur deshalb ab, weil sie ihre Zeitverwendung nüchtern kalkulieren, sondern auch, weil sie ihre eigenen Praktiken und Standards für Haushaltsarbeit nicht als die einzig möglichen ansehen. Ein weiterer entscheidender Punkt ist, dass sie die Leistungen der Dienstleister anerkennen. Pragmatismus und Wertschätzung der Arbeit sind folglich zwei weitere grundlegende Bedingungen, wenn aus dem Bedarf an Haushaltsdienstleistungen ein Arbeitsmarkt entstehen soll, in dem Qualitätsstandards gelten und in dem rechtliche und soziale Mindeststandards gewährleistet sind.

Und als zusätzliche Schubkraft für die mögliche Ausdehnung haushaltsbezogener Dienstleistungen wirkt allemal, dass die Belastung durch volle Erwerbstätigkeit steigt. Gerade viele jüngere Haushalte sehen sich wachsenden Anforderungen gegenüber. Das lässt vermuten, dass sich zunehmend neue - sozusagen unemotionale - Formen des Alltagslebens verbreiten, zu denen dann auch eine Nutzung regulär verfasster Haushaltsdienstleistungen gehört. 


\section{LITERATUR}

Bergmann, J./Geissler, B./Pohlheim, K. (2008): „Haushaltsdienstleistungen - der potenzielle Bedarf “ , Projektbericht, Universität Bielefeld (Fakultät für Soziologie) (Publikation in 2010)

Gather, C./Geissler, B./Rerrich, M. S. (Hrsg.) (2002): Weltmarkt Privathaushalt. Bezahlte Haushaltsarbeit im globalen Wandel, Münster Geissler, B. (2003): Ungeliebt und unverzichtbar: Dienstleistungen im Alltagsleben. Die kulturelle Barriere gegen Haushaltsdienstleistungen, in: Jacobsen, H./Voswinkel, S. (Hrsg.): Dienstleistungsarbeit - Dienstleistungskultur, SAMF-Arbeitspapier 1, S. 97-109

Geissler, B. (2006): Haushalts-Dienstleistungen als informelle Erwerbsarbeit. Neue Ungleichheit oder Ausdifferenzierung des Arbeitsmarkts? in: Arbeit 3, S. 194-205

Geissler, B. (2010): Haushaltsarbeit und Haushaltsdienstleistungen, in: Böhle, F./Voß, G. G./Wachtler, G. (Hrsg.): Handbuch Arbeitssoziologie, Wiesbaden

Gesellschaft für Innovationsforschung und Beratung mbH (GIB) (Hrsg.) (2007): Wachstumspotenzial von privatwirtschaftlich angebotenen haushaltsnahen Dienstleistungen, unveröffl., Berlin

Kaufmann, J.-C. (2005): Mit Leib und Seele. Theorie der Haushaltstätigkeit, Konstanz
Lewis, J. (2004): Auf dem Weg zur "Zwei-Erwerbstätigen-Familie“, in: Leitner, S./Ostner, I./Schratzenstaller, M. (Hrsg.): Wohlfahrtsstaat und Geschlechterverhältnis im Umbruch. Was kommt nach dem Ernährermodell?, Wiesbaden, S. 62-84

Jurczyk, K./ Oechsle, M. (Hrsg.) (2008): Das Private neu denken, Münster

Rerrich, M. S. (1994): Zusammenfügen, was auseinanderstrebt: zur familialen Lebensführung von Berufstätigen. in: Beck, U./Beck-Gernsheim E. (Hrsg.): Riskante Freiheiten. Individualisierung in modernen Gesellschaften, Frankfurt, S. 201-218

Rerrich, M. S. (2006): Die ganze Welt zu Hause. Cosmobile Putzfrauen in privaten Haushalten, Hamburg

Schupp, J./Spieß, K./ Wagner, G. G. (2006): Beschäftigungspotentiale in privaten Haushalten nicht überschätzen, in: DIW-Wochenbericht 4, S. $45-52$

Thiessen, B. (2004): Re-Formulierung des Privaten. Professionalisierung personenbezogener, haushaltsnaher Dienstleistungsarbeit, Wiesbaden Weinkopf, C. (2003): Förderung haushaltsbezogener Dienstleistungen sinnvoll, aber kurzfristige Beschäftigungswirkungen nicht überschätzen, in: Vierteljahreshefte zur Wirtschaftsforschung 1, S. 133-147 